\title{
Seropositivity and risk factors of Brucella infection in small ruminants that had history of recent abortion in the afar region of north-eastern Ethiopia
}

Muluken Tekle ( $\square$ muluken.tekle@aau.edu.et)

Addis Ababa University

Mengistu Legesse

Addis Ababa University

Gobena Ameni

Addis Ababa University

Gezahegne Mamo

Addis Ababa University

Research Article

Keywords: Brucellosis, Seropositivity, mRBPT, cELISA, small ruminants, Ethiopia

Posted Date: December 2nd, 2020

DOI: https://doi.org/10.21203/rs.3.rs-120166/v1

License: (c) (i) This work is licensed under a Creative Commons Attribution 4.0 International License.

Read Full License 


\section{Abstract}

Background: Brucellosis is one of the most important reproductive disease causes abortion and breeding failure in small ruminants and also causes severe systemic diseases in exposed humans. In Ethiopia, several studies of seroprevalence shows the magnitude and distribution of brucellosis both in animals and humans vary in different geographical localities. However, except few studies in Ethiopia all these serological studies was limited to RBPT and CFT, so far not supplemented with a varities of serological tests like ELISA to detect brucella infection, which is increase the likehood of detecting infected individulas and also improve the reliability of epidemiological data for appropriate control strategies. Hence, the present study was conducted in Amibara district of Afar Region, Ethiopia to detect the seropositivity and risk factor of Brucella infection in small ruminants that had history of recent abortion using mRBPT, cELISA and CFT.

Materials and methods: Sera were collected from 226 animals (195 goats and 31sheep) and assessed for seropositivity of Brucella infection using modified Rose Bengal Plate Test (mRBPT), Complement Fixation Test (CFT) and competitive Enzyme Linked Immuno Sorbent Assay (cELISA).

Results : In this study the over all seroprevalence was $12.0 \%$ (27 out of 226 ), $7.5 \%$ (17 out of 226 ) and $26.6 \%$ (60 out of 226) by mRBPT, CFT and cELISA, respectively. Out of 27 sera which were reactive by mRBPT, 17 (63.0\%) were also reactive by (CFT). Out of the 17 sera which were reactive by CFT and mRBPT, 14 (82.4\%) were reactive by cELISA. Out of the 29 sera which were non-reactive both by mRBPT and CFT, 10 (34.5\%) were found to be reactive by CELISA. Out of the 226 sera which were tested both by mRBPT and cELISA, 20 (8.9\%) were reactive by both tests, while 159 (70.4\%) were non-reactive by both tests. The percentage of test agreement (79.2\%) between mRBPT and cELISA was poor $(k=0.353)$. A high seropositivity for Brucella infection was significantly associated with the presence of retained placenta in the study animals (adjusted $\mathrm{OR}=2.2,95 \% \mathrm{Cl}, 1.1-4.4, \mathrm{P}=0.030$ ) as detected by cELISA.

Conclusion: The findings of this study could suggest that brucellosis is main cause of abortion and breeding failure in small ruminants that had histry of recent abortion in the pastoral communities' andwarrants the need for proactive measures to reduce its economic impact and risk of zoonotic transmission. This study indicates that cELISA based seroepidemiological survey increase the likehood of detecting infected individulas of brucellosis and also would be useful to provide reliable evidence for Brucella infection in small ruminants compared to mRBPT.

\section{Introduction}

Brucellosis is a common disease in the tropical and subtropical countries, and causes an enormous economic losses due to it causes abortion and breeding failure in small ruminants and also it affects the health of livestock and diminishes their products [1-2]. It has also been considred as the commonest reemerging zoonotic disease in many areas of the world [3-6]. However, the magnitude and distribution of brucellosis both in animals and humans vary in different giograpical localities [7]. Hence, extensive 
epidemiological survey of brucellosis in humans and animals in different settings using reliable diagnostic methods would be useful to provide reliable epidemiological data.

Bacteriological isolation using culture method, which is a reliable method for the diagnosis of Brucella infection in animals and human requires advanced laboratory, trained manpower and high biosafety suitations. In addition, culture method is not feasible to carry out an epidemiological study of brucellosis [8]. Hence, serological tests such as Rose Bengal Plate Test (RBPT), Complement Fixation Test (CFT) and Enzyme Linked Immuno sorbent Assay (ELISA) in a single or combination are commonly used for the secreening of Brucella infection [8]. Among others, RBPT with or without CFT is the most commnoly used for the screening of Brucella infection in many resource limitted countries including Ethiopia [9-12]. However, RBPT has been criticized for its drawbacks such as false-positive results due to cross-reactivity with other bacteria $[13,14]$.

Several studies have also suggested that ELISA is more effective for a sero-epidemiological survey of brucellosis as compared to RBPT and good diagnostic results have been obtained in sheep and goats with indirect (I-) or competitive (C-) enzymelinked immunosorbent assays (ELISAs) using various antigens, but generally the ELISAs that use antigens with a high content of smooth lipopolysaccharide (sLPS) are the most useful [15-19 and 41].

In Ethiopia, several seroprevalence studies of brucellosis have shown the occurrence of brucellosis among livestock using mRBPT and CFT [20-23]. Nevertheless, few studies have used ELISA to assess the seroprevalence of brucellosis in animals $[22,24]$. Previous studies showed that cELISA is highly specific compared to indirect ELISA and mRBPT for the diagnosis of brucellosis in animals $[18,25$, and $26]$. Therefore, the present study was conducted in the afar region of north-eastern Ethiopia to detect the seropositivity and risk factor of Brucella infection in small ruminants that had history of recent abortion using mRBPT, cELISA and CFT.

\section{Methods}

\section{Study area and animal population}

This study was conducted in Amibara district of the Afar Region, north-east Ethiopia. Detailed description of the study area has been given elsewhere [27]. In Ethiopia to date, no brucellosis vaccination has been undertaken in animals [24, 28, and 29].

\section{Sample size estimation}

With the assumption of $16 \%$ seroprevalence of Brucella infection in small ruminants in the study area [24] 5\% precision and 95\% confidence level, about 207 animals were intended to be included in the study. 


\section{Study animals and procedure of data collection}

A community based cross-sectional study was conducted from October, 2015 to April, 2016. A house-tohouse survey was conducted to include goats and/or sheep that had history of recent abortion (abortion occured in last 30 days at the time of data collection) were included in the study. After the aim of the survey had been explained, the owners were interviewed using structured questionnaire regarding history of abortion, the duration of abortion, age of the animal, frequency of abortion and retained placenta. Then, about $3 \mathrm{ml}$ blood samples was collected from each animal, serum was separated and stored at $-20^{\circ} \mathrm{C}$ until processed for serological analysis.

\section{Serological tests}

All sera were screened using mRBPT and cELISA as per the manufacturers' instruction

(Svanova, Brucella-ab c-ELISA Uppsala Business Park. Rapsgatan 7, 75174 Uppsala, Sweden). All sera found positive by mRBPT were further tested by CFT. In addition, 29 randomly selected sera which were negative by mRBPT were also tested by CFT.

\section{Data Analysis}

Data were entered into EpiData Software v.3.1 and analyzed using Stata version 11. Frequencies and percentages were used to summarise baseline characteristics of the study animals and the seroprevalence of brucellosis as diagnosed using mRBPT, CFT and cELISA. Univariable and multivariable logistic regression analyses were used to assess the effect of each of the independent variables (such as age, history of abortion and retained placenta). A p-value less than 0.05 were considered statistically significant. Agreement between the tests was assessed using Cohen's Kappa $(\mathrm{k})$ coefficient. $\mathrm{K}$ values greater than 0.75 between 0.4 and 0.75 and less than 0.4 were considered excellent, fair and poor agreement, respectively.

\section{Ethics approval and consent to participate}

The study protocol was approved by the Institutional Review Board (IRB) of the College of Veterinary Medicine and Agriculture (CVMA), Addis Ababa University. The purpose of the study was explained to all small ruminant owners who participated in the study and verbal consent was obtained from the animal owners before collecting samples from small ruminants. The IRB approved the verbal consent from owners would be enough for blood sample collection from aborted goats since the procedure was taken as a routine clinical procedure to confirm Brucella diagnosis.

\section{Results}

\section{Baseline characteristics of the study animals and seroprevalence of brucellosis}


According to the report by the owners, 98 (43.4\%) animals had abortion history for two or more times and about half $(49.6 \%)$. More than half $(52.7 \%)$ of the study animals had retained placenta during the data collection.Table 1 shows the baseline characteristics of the 226 study animals and seroprevalence of brucellosis as detected by RBPT and CFT. Out of the total 226 animals, 27 (12.0\%) were positive for Brucella infection by the mRBPT. Out of those 27, 17 (63.0\%) were positive for Brucella infection by the CFT. None of the 29 animals which were negative by mRBPT found positive by CFT.

Table 1. Baseline characteristics of the study animals and seropositivity for Brucella infection by mRBPT and CFT

\begin{tabular}{|c|c|c|c|c|}
\hline \multirow[t]{3}{*}{ Characterstics } & \multicolumn{2}{|l|}{$\mathrm{RBPT}$} & \multicolumn{2}{|l|}{$\mathrm{CFT}$} \\
\hline & Number tested (\%) & Number & Number tested (\%) & Number \\
\hline & & Positive (\%) & & Positive (\%) \\
\hline \multicolumn{5}{|l|}{ Animal species } \\
\hline Goat & 195 (86.3) & $26(13.3)$ & $50(25.6)$ & $16(32.0)$ \\
\hline Sheep & $31(13.7)$ & $1(3.2)$ & $6(19.4)$ & $1(16.7)$ \\
\hline \multicolumn{5}{|l|}{ Age in years } \\
\hline $2.0-3.9$ & $82(36.3)$ & $6(7.3)$ & $15(18.3)$ & $5(33.3)$ \\
\hline $4.0-5.9$ & $89(39.4)$ & $9(10.1)$ & $26(29.2)$ & $4(15.4)$ \\
\hline$\geq 6.0$ & $55(24.3)$ & $12(21.8)^{*}$ & $15(27.3)$ & $8(53.3)^{*}$ \\
\hline \multicolumn{5}{|l|}{ Parity } \\
\hline None & $46(20.4)$ & $6(13.0)$ & $6(13.0)$ & $6(100)^{*}$ \\
\hline $1-2$ & $87(38.7)$ & $13(14.9)$ & $24(27.6)$ & $6(25.0)$ \\
\hline$\geq 3$ & $92(40.9)$ & $8(8.7)$ & $25(27.2)$ & $5(20.0)$ \\
\hline \multicolumn{5}{|c|}{ Stage of abortion } \\
\hline First month & $34(15.0)$ & $1(2.9)$ & $1(2.9)$ & $1(100)$ \\
\hline Second month & $80(35.4)$ & $1(1.3)$ & $11(13.8)$ & $0(0)$ \\
\hline Third month & $112(49.6)$ & $25(22.3) *$ & $44(39.3)$ & $16(36.4)$ \\
\hline \multicolumn{5}{|c|}{ Frequency of abortion } \\
\hline Once & $128(56.6)$ & $11(8.6)$ & $32(25.0)$ & $8(25.0)$ \\
\hline$\geq 2$ times & $98(43.4)$ & $16(16.3)$ & $24(24.5)$ & $9(37.5)$ \\
\hline \multicolumn{5}{|c|}{ Retained placenta } \\
\hline Absent & $107(47.3)$ & $1(0.9)$ & $12(11.2)$ & $0(0)$ \\
\hline Present & $119(52.7)$ & $26(21.9) *$ & $44(37.0)$ & $17(38.6)^{*}$ \\
\hline
\end{tabular}

* Pearson's chi-square test was statistically significant at $\mathrm{p}<0.05$

On the other hand, 60 (26.6\%) animals were found positive for Brucella infection by cELISA. Out of the 27 animals which were positive for Brucella infection by mRBPT, 7(25.9\%) were negative by cELISA. Out 
of the 17 animals which were positive for Brucella infection by CFT and mRBPT, 14 (82.4\%) were positive by cELISA.

In general, cELISA revealed a high seropositivity for Brucella infection in the study animals with retained plancenta compared to animals without retained placenta $\left(34.5 \%\right.$ versus $\left.17.8 \%, x^{2}=8.05, p=0.005\right)$. In bivariate logestic regression analysis, age over 6 years (Crude $\mathrm{OR}=2.3,95 \% \mathrm{Cl}, 1.1-5.1, \mathrm{P}=0.030$ ) and retained placenta (crude $\mathrm{OR}=2.4,95 \% \mathrm{Cl}, 1.3-4.5, \mathrm{P}=0.005$ ) were significantly assocated with a high seropositivity for Brucella infection as detected by cELISA. In multivariable logistic regression analysis, only retained placenta (adjusted $\mathrm{OR}=2.2,95 \% \mathrm{Cl}, 1.1-4.4, \mathrm{P}=0.030$ ) was significantly associated with a high seropositivity for Brucella infection (Table 2).

Table 2. Association between baseline characteristics of the study animals and seropositivity for Brucella infection as detected by cELISA

\begin{tabular}{|c|c|c|c|c|}
\hline iable & Number (\%) & $\begin{array}{l}\text { Number } \\
\text { Positive (\%) }\end{array}$ & $\begin{array}{l}\text { Adjusted OR } \\
(95 \% \text { CI })\end{array}$ & $P$ value \\
\hline \multicolumn{5}{|l|}{3} \\
\hline :-3.9 years & $82(36.3)$ & $16(19.5)$ & Reference & \\
\hline$=-5.9$ years & $89(39.4)$ & $24(27.0)$ & $1.38(0.54-3.52)$ & 0.505 \\
\hline$\geq 6$ years & $55(24.3)$ & $20(36.4)$ & $1.92(0.66-5.57)$ & 0.230 \\
\hline \multicolumn{5}{|l|}{ ity } \\
\hline None & $46(20.4)$ & $10(21.7)$ & Reference & \\
\hline $1-2$ & $87(38.7)$ & $23(26.4)$ & $1.00(0.39-2.56)$ & 0.998 \\
\hline$\geq 3$ & $92(40.9)$ & $27(29.4)$ & $0.99(0.34-2.87)$ & 0.895 \\
\hline \multicolumn{5}{|l|}{ ge of abortion } \\
\hline st month & $34(15.0)$ & 7 (20.6) & Refrence & \\
\hline sond month & $80(35.4)$ & $16(20.0)$ & $0.94(0.33-2.69)$ & 0.906 \\
\hline rd month and above & $112(49.6)$ & $37(33.0)$ & $1.44(0.55-3.77)$ & 0.463 \\
\hline \multicolumn{5}{|l|}{ quency of abortion } \\
\hline ace & $128(56.6)$ & $29(22.7)$ & Reference & \\
\hline times & $98(43.4)$ & $31(31.6)$ & $1.46(0.71-2.98)$ & 0.301 \\
\hline \multicolumn{5}{|l|}{ ained placenta } \\
\hline Absent & $107(47.3)$ & $19(17.8)$ & Reference & \\
\hline Present & $119(52.7)$ & $41(34.5) *$ & $2.17(1.08-4.37)$ & 0.030 \\
\hline
\end{tabular}

\section{Agreement of the tests for the screening of Brucella infection in small ruminants}

Table 3 shows test agreement between mRBPT and cELISA for the screening of Brucella infection in the study animals. Out of the 226 sera which were tested both by mRBPT and cELISA, 20 (8.9\%) were positive by both tests, while 159 (70.4\%) were negative by both tests. Hence, the percentage of agreement $(79.2 \%)$ between mRBPT and cELISA was poor $(k=0.353)$. Out of the total 56 sera which 
were tested both by CFT and CELISA, 14 (25\%) were positive by both tests, while 29 (51.8\%) were negative by both tests, and the percentage of agreement (76.8\%) between cELISA and CFT was also poor ( $\mathrm{k}=$ 0.193).

Table 3. Tests agreement for the sero-diagnosis of Brucella infection in the study animals.

\begin{tabular}{llll}
\hline & \multicolumn{2}{c}{ CFT result } & \\
\cline { 2 - 4 } mRBPT (n=56) & Number Positive & Number Negative & Total number tested \\
\hline Number Positive & 17 & 10 & 17 \\
Number Negative & 0 & 29 & 29 \\
\hline & cELISA result & & \\
\hline mRBPT (n=226) & Number Positive & Number Negative & Total number tested \\
\hline Number Positive & 20 & 7 & 27 \\
\hline Number Negative & 40 & 159 & 199 \\
\hline & CFT result & & Total number tested \\
\hline cELISA (n=56) & Number Positive & Number Negative & 24 \\
\hline Number Positive & 14 & 10 & 32 \\
\hline Number Negative & 3 & 29 &
\end{tabular}

\section{Discussion}

The present study determined the overall seroprevalence of brucellosis in sheep and goats with history of recent abortion is between $12 . \%$ and $7.5 \%$ with mRBPT alone and using combined mRBPT CFT tests, respectively. The observed seroprevalence is higher than seroprevalence reported in small ruminants in other areas of Ethiopia using CFT [21,31], but relatively lower than previously reported overall seroprevalence of brucellosis in small ruminants using CFT in the Afar Region [28,29].

In this study, we assessed a seroprevalence of brucellosis in small ruminants with history of recent abortion by mRBPT and cELISA. The seroprevalence of brucellosis was $12.0 \%$ in the study animals as detected by mRBPT which is similar to a recently reported seroprevalene of brucellosis in small ruminants in other districts of the Afar Region [28,29]. Another study in Afar Region showed a low seroprevalence (3.1\%) of brucellosis in small ruminants using mRBPT [24]. Study in other pastoral areas of Ethiopia also revealed relatively a low seroprevalence (8.5\%) of the diseases using mRBPT [21]. On the other hand, Tadeg et al. [30] reported a high seroprevalence of brucellosis (17.4\%) using mRBPT in small ruminants in other area of the Afar Region. 
In this study, cELISA revealed a high seroprevalence for Brucella infection in the study animals as compared to that of mRBPT $\left(26.6 \%\right.$ VS $\left.12.0 \%, x^{2}=35.5, p<0.001\right)$. Previous studies in Ethiopia also showed a significantly high seroprevalence of brucellosis in small ruminants using iELISA as compared to mRBPT [24]. Similar to the findings of the present study, previous studies in small ruminants suggested that ELISA based test is more effective for the serological based survey of brucellosis as compared to mRBPT [16-19].

In this study, the agreement between mRBPT and cELISA for the serodiagnosis of Brucella infection in small ruminants was poor. However, previous study in Ethiopia revealed a fair agreement between mRBPT and indirect ELISA for the sero-diagnosis of Brucella infection in small ruminants [24]. On the other hand, study in Rwanda has shown an excellent agreement between mRBPT and CELISA ( $K=0.92)$ for the serodiagnosis of Brucella infection in cattle [32]. The low agreement between mRBPT and cELISA in the present study was attributed to the high seroprevalence of brucellosis detected by the cELISA which might be due to the ability of this ELISA based technique to detect low level of antibody even at early stage of infection $[17,33]$.

In this study, some sera which were positive either by mRBPT or both by MRBPT and CFT were found negative by cELISA. In recent studies conducted in small ruminants elsewhere a considerable number of sera which were positive by mRBPT were found negative by ELISA [34-36]. In this study, a high seroprevalence for Brucella infection was detected in those animals with retained placenta which is similar to the results of previous studies elsewher [37-40]. A review on clinical features of brucellosis also showed that placenta retention is one of the main clinical signs of $B$. melitensis infection in aborted small ruminants [1].

In order to evaluate the reliability of the mRBPT and/or cELISA for the serodiagnosis of brucellosis, gold standard (bacteria culture) need to be used. However, in this study, the seroprevalence of brucellosis in animals with a history of recent abortion was assessed and compared using mRBPT and cELISA without using a gold standard like several previous studies and this could be one of the limitations of this study.

\section{Conclusion}

In this study demonstrated high seropositivity of brucella infection in small ruminants with the history of recent abortion could suggest that brucellosis is main responsible cause of abortion and breeding failure in small ruminants of pastoral communities. Beside this a high seropositivity of brucella infection in small ruminants was detected by cELISA as compared to mRBPT. Moreover, seropositivity for Brucella infection was significantly associated with retained placenta. Hence, this finding suggest that cELISA based test is more effective for the serological based survey of brucellosis in small ruminants as compared to mRBPT in the present study area though additional confirmational studies are important. And also this finding warrants appropriate control strategies to reduce its economic impact and risk of zoonotic transmission of the disease in the study area. 


\section{Declarations}

Competing Interests:

The authors declared that they have no competing interests.

\section{Acknowledgements}

Afar Pastoral Regional State of Amibara district, animal owners and Melka Were Agriculture Research Center are highly acknowledged. This Study was financially suported by the Institute of Tropical Medicine (ITM-Belgium). The funding organization had no any role in the design of the study and collection, analysis, and interpretation of data and in writing the manuscript.

\section{References}

1. Megid J, Mathias LA, Robles CA. Clinical Manifestations of Brucellosis in Domestic Animals and Humans. Vet Sci J. 2010; 4:119-

2. Rossetti CA, Arenas AM, Maurizio E. Caprine brucellosis: A historically neglected disease with significant impact on public health. PLoS Negl Trop Dis. 2017; 11:56-92.

3. Pal M, Gizaw F, Fekadu F, Alemayehu G, Kandi V. Public Health and Economic Importance of Bovine Brucellosis: An Overview. Amer J Epidemiol Infect Dis. 2017; 5: 27-

4. Donev D, Karadzovski Z, Kasapinov B, Lazarevik V. Epidemiological and public health aspects of brucellosis in the Republic of Mecedonia. Biolog Med Scie. 2010; 1:33-

5. Liu Q, Cao L, Zhu Major emerging and re-emerging zoonoses in China: a matter of global health and socioeconomic development for 1.3 billion. Inter J Infect Dis. 2014; 25: 65-72.

6. Seleem MN, Boyle SM, Sriranganathan N. Brucellosis: a re-emerging zoonosis. Vet Microbiol. 2010; 140: $392-$

7. Dean AS, Crump L, Greter H, Hattendorf J, Schelling E, Zinsstag J. Clinical manifestations of human brucellosis: a systematic review and meta-analysis. PLoS Negl Trop Dis. 2012;6(12):e1929.

8. Nielsen K, Yu Serological diagnosis of brucellosis. Prilozi. 2010;31(1):65-89.

9. Tadesse G. Brucellosis Seropositivity in Animals and Humans in Ethiopia: A Meta-analysis. PLoS Negl Trop Dis. $2016 ; 10(10): \mathrm{e} 0005006$

10. Swai MS, Schoonman L. The Use of Rose Bengal Plate Test to Asses Cattle Exposure to Brucella Infection in Traditional and Smallholder Dairy Production Systems of Tanga Region of Tanzania. Vet Med Int. $2010 ; 2010$, 8 pages.

11. Saxena N, Singh BB, Saxena HM. Brucellosis in sheep and goats and its serodiagnosis and epidemiology. Int J Curr Microbiol App Sci. 2018; 7(1) : 1848-1877.

12. Chisi SL, Marageni Y, Naidoo P, Zulu G, Akol GW, Van Heerden H. An evaluation of serological tests in the diagnosis of bovine brucellosis in naturally infected cattle in KwaZulu-Natal province in South 
Africa. J S Afr Vet Assoc. 2017; 88(0):e1-e7.

13. Nielsen $K^{1}$, Smith $P$, Yu W, Nicoletti P, Jungersen G, Stack J, Godfroid J. Serological discrimination by indirect enzyme immunoassay between the antibody response to Brucella sp. and Yersinia enterocolitica 0:9 in cattle and pigs. Vet Immunol Immunopathol. 2006; 109(1-2):69-78.

14. Chart $\mathrm{H}$, Okubadejo OA, Rowe B. The serological relationship between Escherichia coli 0157 and Yersinia enterocolitica 09 using sera from patients with brucellosis. Epidemiol Infect. 1992;108(1):77-85.

15. Gall D, Nielsen K. Serological diagnosis of bovine brucellosis: a review of test performance and cost comparison. Rev Sci Tech. 2004 ;23(3):989-1002.

16. Nielsen K, Smith P, Yu WL, Elmgren C, Halbert G, Nicoletti P, et al. Validation of a second generation competitive enzyme immunoassay (CELISA) for the diagnosis of brucellosis in various species of domestic animals. Vet Immunol Immunopathol. 2008;125(3-4):246-50.

17. Perrett $L^{1}{ }^{1}$, McGiven JA, Brew SD, Stack JA. Evaluation of competitive ELISA for detection of antibodies to Brucella infection in domestic animals. Croat Med J.2010;51(4):314-9.

18. Ahmed M, Islam MDA, Khatun MM, Baek BK. Evaluation of four Serological Tests for the Detection of Brucellosis in Goats and Cattle under the Field Condition of Bangladesh. Asian J Biolog Scie, 2011 : 4: 477-482.

19. Saxena N, Singh BB, Gill JPS, Aulakh RS.Frequency of Occurance of Brucellosis in Goats in Ludhiana District of Punjab State of India. Microbiol Research J Int 2017; 21(6): 1-7

20. Ashenafi F, Teshale S, Ejeta G, Fikru R, Laikemariam Y. Distribution of brucellosis among small ruminants in the pastoral region of Afar, eastern Ethiopia. Rev Sci Technol. 2007; 26: 731-739.

21. Tsehay H, Getachew G, Morka A, Tadesse B, Eyob seroprevalence of brucellosis in small ruminants in pastoral areas of oromia and Somalia regional states, Ethiopia. J Vet Med. 2014; 6(11): 289-294.

22. Tschopp R, Bekele S, Moti T, Young D, Aseffa A. Brucellosis and bovine tuberculosis prevalence in livestock from pastoralist communities adjacent to Awash National Park, Ethiopia. Prev Vet Med. 2015; 120(2):187-94.

23. Sintayehu G, Melesse B, Abayneh D, Sintayehu A, Melaku S, Alehegne W, et al.. Epidemiological survey of brucellosis in sheep and goats in selected pastoral and agro-pastoral lowlands of Ethiopia. Rev Sci Tech Int Epiz. 2015; 34 (3):1-25.

24. Teshale $S$, muhie $Y$, dagne $A$, kidanemariam A.Seroprevalence of small ruminant brucellosis in selected districts of Afar and Somali pastoral areas of Eastern Ethiopia: the impact of husbandry practice. Revue Méd Vét. 2006; 157: (11) 557-563.

25. Nielsen K, Gall D, Smith P, Balsevicius S, Garrido F, Ferrer MD.Comparison of erological tests for the detection of ovine and caprine antibody to Brucella melitensis. Rev Sci Tech. 2004; 23 (3):979-87.

26. Etman RH, Barsoum SA, Ibrahim IGA, El-Ashmawy WR Abou-Gazia KH. Evaluation of efficacy of some serological tests used for diagnosis of brucellosis in cattle in Egypt using latent class analysis . Sokoto J VetSci2014; 12(2):1-7. 
27. Mamo G, Abebe F, Worku Y, Hussein N, Legesse M, Tilahun G, Medhin G, Bjune G, Ameni G. Bovine tuberculosis and its associated risk factors in pastoral and agro-pastoral cattle herds of Afar Region, Ethiopia. J Vet Med Ani Health. 2013; 5(6):171-

28. Tegegn AH, Feleke A, Adugna W, Melaku SK. Small Ruminant Brucellosis and Public Health Awareness in Two Districts of Afar Region, Ethiopia. J Vet Sci Technol. 2016 ; 7:335.

29. Adugna W, Tessema TS, Keskes S. Sero-prevalence of small ruminants' brucellosis in four districts of Afar National Regional State, Northeast Ethiopia. J Vet Med Anim Health. 2013; 5(12) 358-364

30. Tadeg WM , Gudeta FR, Mekonen TY, Asfaw YT, Birru AL Reda AA. Seroprevalence of small ruminant brucellosis and its effect on reproduction at Tellalak District of Afar region, Ethiopia. $J$ Vet Med Anim Health 2015 ; 7(4), 111-116.

31. Dulo F. Seroprevalence of Caprine Brucellosis and Its Associated Risk Factor in Mirab Abay district, South Eastern Ethiopia. J Natu Sci Research 2017;7 (9): ISSN 2224-3186

32. Manishimwe R, Ntaganda J, Habimana R, Nishimwe K, Byukusenge M, et al. Comparison between Rose Bengal Plat Test and Competitive Enzyme Linked Immunosorbent Assay to Detect Bovine Brucellosis in Kigali City, Rwanda. J Veterinar Sci Technol. 2015 ; 6:211. doi:10.4172/21577579.1000211

33. Bishaif R, Galli R. Enzyme-Linked Immunosorbent Assay for Detection of Antibodies to Influenza A and B and Parainfluenza Type 1 in Sera of Patients. J Clin Microbiol, 1978; 8: 648-656.

34. Ogugua AJ, Akinseye VO, Ayoola MC, Oyesola OO, Shima FK, Tijjani AO, Seroprevalence and risk factors of brucellosis in goats in selected states in Nigeria and the public health implications. Afr J Med Sci. 2014 ;43(Suppl 1):121-129.

35. Sadhu DB, Panchasara HH, Chauhan HC, Sutariya DR, Parmar VL, Prajapati HB. Seroprevalence and comparison of different serological tests for brucellosis detection in small ruminants. Vet World $2015 ; 8(5): 561-566$.

36. Mohamed EMA, Elfadi AAM, El-Sanousi EM, Ibrahaem HH, Mohamed-Noor SET, Abdella MA, Shuaib YA. Seroprevalence and risk factors of caprine brucellosis in Khartoum state, Sudan. Vet World 2018; 11(4) 511-518.

37. Islam MA, Samad MA, Rahman AKMA. Risk factors associated with prevalence of brucellosis in Black Bengal goats in Bangladesh. Bangl J vet Med 2010; 8 (2) 141-147.

38. Kelkay MZ, Gugsa G, Hagos Y, Taddelle H. Seroprevalence and associated risk factors for Brucella seropositivity among small ruminants in Tselemti districts, Northern Ethiopia. J Vet Med Animal Health $2017 ; 9$ (11):320-326.

39. Sharma V, Sharma HK, Ganguly S, Berian S, Malik MA. Seroprevalence studies of brucellosis among goats using different serological tests. J Entomol Zool Stud 2017 ; 5 (2) : 1512-1516.

40. Ahasan MS, Rahman MS, Rahman AK, Berkvens D. Bovine and Caprine Brucellosis in Bangladesh: Bayesian evaluation of four serological tests, true prevalence, and associated risk factors in household animals. Trop Anim Health Prod. 2017 ; 49(1):1-11. 
41. Office International des Epizooties (OIE). Bovine Brucellosis; caprine and ovine brucellosis and porcine brucellosis. In: World assembly of delegates of the OIE chapter 2.4.3. Paris: OIE Terrestrial Manual; 2009. p. 1- 35. 